\title{
TEMPORAL DYNAMICS OF BRAIN ACTIVATION DURING THREE CONCEPT GENERATION TECHNIQUES
}

\author{
Milovanovic, Julie (1); \\ Hu, Mo (2); \\ Shealy, Tripp (2); \\ Gero, John (3) \\ 1: UMR AAU-CRENAU, Graduate School of Architecture of Nantes, Nantes, France; \\ 2: Department of Civil and Environmental Engineering, Virginia Tech, Blacksburg, USA; \\ 3: Department of Computer Science and School of Architecture, University of North Carolina
}

\begin{abstract}
The research presented in this paper explores features of temporal design neurocognition by comparing regions of activation in the brain during concept generation. A total of 27 engineering graduate students used brainstorming, morphological analysis, and TRIZ to generate concepts to design problems. Students' brain activation in their prefrontal cortex (PFC) was measured using functional near-infrared spectroscopy (fNIRS). Temporal activations were compared between techniques. When using brainstorming and morphological analysis, highly activated regions are consistently situated in the medial and right part of the PFC over time. For both techniques, the temporal neuro-physiological patterns are similar. Cognitive functions associated to the medial and right part of the PFC suggest an association with divergent thinking and adaptative decision making. In contrast, highly activated regions over time when using TRIZ appear in the medial or the left part of the prefrontal cortex, usually associated with goal directed planning.
\end{abstract}

Keywords: Design cognition, Design process, Design engineering, Design neurocognition, Neuroimaging

Contact:

Milovanovic, Julie

AAU-CRENAU

Graduate School of Architecture

France

julie.milovanovic@crenau.archi.fr 


\section{INTRODUCTION}

Generating concepts is an important step in the engineering design process (Hay et al., 2017). Concept generation relies on cognitive activities that mobilize a large range of cognitive functions (Heilman et al., 2003). Designing evolves over time and time dynamics appear as an essential aspect of that cognitive activity. This temporal dimension relates to the situatedness of design (Gero, 1999; Schön, 1983) both at a contextual level (design artifact) and personal level (designer). Many techniques are available to help designers generate concepts, such as brainstorming, morphological analysis and TRIZ (Smith, 1998). These techniques vary in intuitiveness, motivation and structuredness in their implementation (Gero et al., 2013). The specifics of each concept generation technique imply that designers will rely on different cognitive processes to generate concepts depending on the technique used.

In this paper, we explore that dimension using methods adopted from neuroscience. We examine how the use of different concept generation techniques affect activation in designers' prefrontal cortex (PFC). The purpose of this research is to measure the dynamic neurocognitive activation in the PFC. The aim is to understand how the different structuredness of techniques for concept generation over time lead to different dynamic patterns of high activation in the PFC. The research question is how does activation in the PFC change over time when generating concepts using techniques with different levels of structuredness?

The emergent use of neuroimaging techniques to study design cognition using diverse tools and methods from neurosciences (Borgianni \& Maccioni, 2020) such as fMRI (Alexiou et al., 2011; Fu et al., 2019; Goucher-Lambert et al., 2019; Hay et al., 2019), fNIRS (Shealy et al., 2020) or EEG (Vieira et al., 2020) aims at better interpretation of the relation between designers' minds and brains (Gero \& Milovanovic, 2020). Knowledge of brain activations during designing has the potential to provide objective measurement of design cognition more generally. The exploration of mapping between cognitive design processes and neurological measurements can help determine whether designing is a set of unique mental activities or a unique combination of generic mental activities. The study presented in this paper is a step in that direction. A more complete understanding of the mental activities during design will require more research. This study begins to provide a foundation by making initial connections between design techniques and the patterns of mental activities in the brain when using these techniques.

\section{BACKGROUND}

\subsection{Concept generation techniques}

Previous design cognition studies compared three techniques to generate concepts: brainstorming, morphological analysis and TRIZ (Gero et al., 2013). Brainstorming is more intuitive than morphological analysis and TRIZ, and is inner sense driven compared to TRIZ's problem driven motivation (Table 1). Brainstorming is unstructured whereas morphological analysis and TRIZ follow a set of steps (Altshuller, 1997). Different techniques lead to varying cognitive responses (Chulvi et al., 2012; Gero et al., 2013; Tang et al., 2012). Structured techniques like morphological analysis and TRIZ encourage designers to first think through the problem, to decompose the problem into manageable sub-problems before considering potential solutions (Gero et al., 2013).

Table 1. Characteristics of concept generation techniques

\begin{tabular}{|l|l|l|l|}
\hline Techniques & \multicolumn{1}{|c|}{ Brainstorming } & Morphological analysis & \multicolumn{1}{c|}{ TRIZ } \\
\hline Intuitiveness & Intuitive & Intuitive & Logical \\
\hline Motivation & Inner sense driven & Problem-driven & Problem-driven \\
\hline Structure & Unstructured & Partially structured & Structured \\
\hline Steps & $\begin{array}{l}\text { 1) Generate as many } \\
\text { solutions as possible } \\
\text { and suspend evaluation }\end{array}$ & $\begin{array}{l}\text { 1) Define and decompose } \\
\text { the problem } \\
\text { 2) Generate multiple sub- } \\
\text { solutions to each sub- } \\
\text { problem } \\
\text { 3) Generate final solutions }\end{array}$ & $\begin{array}{l}\text { 1) Define the problem } \\
\text { 2) Search for standard } \\
\text { engineering parameters } \\
\text { 3) Search for standard } \\
\text { catalogued solutions } \\
\text { 4) Generate final solutions }\end{array}$ \\
\hline
\end{tabular}




\subsection{Design neuro-cognition}

Concept generation techniques impact temporal organization during design. Structured techniques, like TRIZ, can lead to varying patterns of neurocognitive activation in response to the order of tasks and steps during concept generation (Alexiou et al., 2011; Hu \& Shealy, 2019; Shealy et al., 2018).

The active use of distinct cognitive functions during design is observable in the brain (Goel, 2014; Goldschmidt, 2016; Laspia et al., 2019; Shealy et al., 2017) (Table 2). For example, the left prefrontal cortex (PFC) is highly activated during brainstorming early in the process, but when using morphological analysis, the left PFC becomes more activated towards the end of the process (Shealy \& Gero, 2019). Phases of problem solving appear in brain activation patterns, switching from the left dorsolateral PFC in earlier stages (information gathering and problem representation) to the right dorsolateral PFC for later stages (mental transformation and planning) (Ruh et al., 2012). The activation associated with cognitive flexibility in the PFC also varies when using brainstorming, morphological analysis, and TRIZ, suggesting different mental workload (Shealy et al., 2020). This dynamic increase in activation in the left PFC or right PFC in relation to time and generation techniques might suggest mapping patterns between cognitive design processes and neurological activation.

Table 2. Cognitive functions associated with activation in the prefrontal cortex (PFC)

Part of the brain

Associated functions

\begin{tabular}{|c|c|}
\hline $\begin{array}{l}\text { Prefrontal cortex } \\
(P F C)\end{array}$ & $\begin{array}{ll}\text { - } & \text { Planning and executing (Dietrich, 2004) } \\
\text { - } & \text { Sustaining focused attention, information selection and performing } \\
\text { executive functions (Lara \& Wallis, 2015) }\end{array}$ \\
\hline Right prefrontal cortex & $\begin{array}{l}\text { - } \quad \text { Divergent thinking (Aziz-Zadeh et al., 2013; Goel \& Grafman, 2000; } \\
\text { Wu et al., 2015) } \\
\text { - Strong synchronization in the right PFC is associated with higher } \\
\text { originality in solution generation (Fink et al., 2009) }\end{array}$ \\
\hline $\begin{array}{l}\text { Right dorsolateral } \\
\text { prefrontal cortex } \\
(\text { DLPFC) }\end{array}$ & 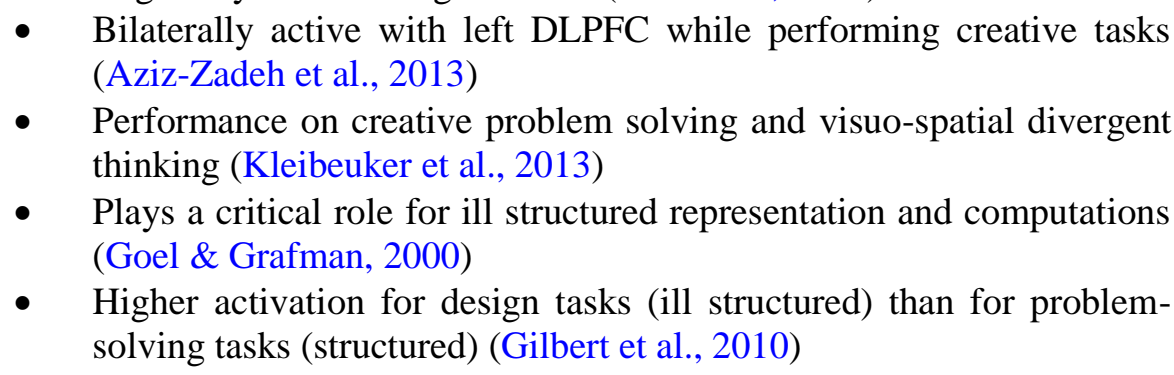 \\
\hline $\begin{array}{l}\text { Right ventrolateral } \\
\text { prefrontal cortex } \\
(V L P F C)\end{array}$ & $\begin{array}{l}\text { - Evaluating problems rather than solving it (Aziz-Zadeh et al., 2009) } \\
\text { - Support the generation of alternative hypothesis to explore the } \\
\text { problem space (Goel \& Vartanian, 2005) }\end{array}$ \\
\hline Left prefrontal cortex & $\begin{array}{l}\text { - } \quad \text { Rule-based design and goal-directed planning (Aziz-Zadeh et al., } \\
\text { 2013) } \\
\text { - } \quad \text { Making analytical judgment (Gabora, 2010) } \\
\text { - Control judgment (Luft et al., 2017) }\end{array}$ \\
\hline $\begin{array}{l}\text { Left dorsolateral } \\
\text { prefrontal cortex } \\
(\text { DLPFC) }\end{array}$ & $\begin{array}{l}\text { - Bilaterally active with right DLPFC while performing creative tasks } \\
\text { (Aziz-Zadeh et al., 2013) } \\
\text { - } \quad \text { Goal directed planning of novel solutions (Aziz-Zadeh et al., 2013) }\end{array}$ \\
\hline $\begin{array}{l}\text { Medial prefrontal } \\
\text { cortex ( } m P F C)\end{array}$ & 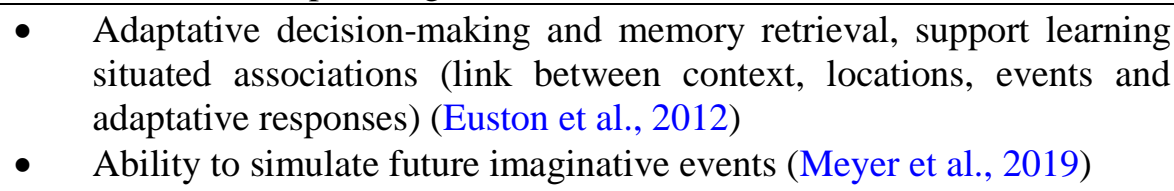 \\
\hline
\end{tabular}

\section{METHODS}

Twenty-seven engineering students (all right-handed, 22-26 years old) participated in the study. All participants had taken courses in engineering design and were familiar with brainstorming. They were given instructions about morphological analysis and TRIZ. Participants received three design tasks: designing a device to assist the elderly with raising and lowering windows, designing an alarm clock for the hearing impaired, and designing a kitchen measuring tool for the blind. The design tasks were 
previous validated and used in prior studies (Gero et al., 2013). The design tasks were similar in scope but did not address the same design problem. This was meant to help prevent recall between design tasks. Participants were instructed to use one of three techniques to develop solutions for each of the problems. The order of techniques and problems were assigned randomly. Each student generated concepts for all three problems using one of the three techniques without time limit constraints.

A neuroimaging technique called functional near-infrared spectroscopy (fNIRS) was used to capture the brain activation in the PFC. fNIRS measures the change of oxy-hemoglobin (HbO) and deoxy-hemoglobin in the brain blood flow. Figure 1 shows the placement of the light sensors in the prefrontal cortex (PFC) in the experiment. Multiple sub-regions of PFC are covered, including the dorsolateral prefrontal cortex (DLPFC: channels 1, 2, 3, 9, 10 in the right hemisphere, and channels 5, 6, 7, 13, and 14 in the left hemisphere), ventrolateral prefrontal cortex (VLPFC: channels 16 and 17 in the right hemisphere, and channels 21 and 22 in the left hemisphere), orbitofrontal cortex (OFC: channel 18 in the right hemisphere, and channel 20 in the left hemisphere), and medial prefrontal cortex (mPFC: channels 4, 11, 12 and 19).

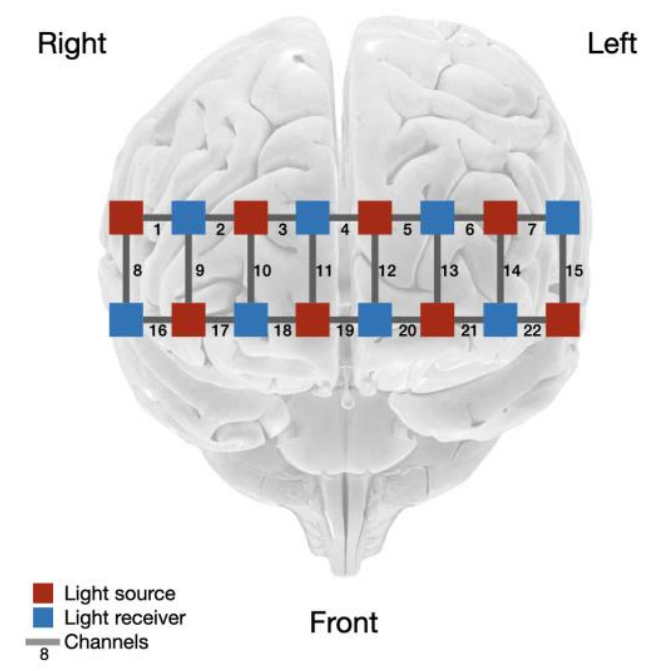

Figure 1. Sensor configuration (Base of brain image copyright (C) Society for Neuroscience (2017))

We processed the fNIRS raw data using a bandpass filter $(0.01-0.1 \mathrm{~Hz}$, third-order Butterworth filter) and independent component analysis (ICA, with a coefficient of spatial uniformity of 0.5 ) to remove noise and motion artifacts (Santosa et al., 2017). $\mathrm{HbO}$ have higher amplitudes and sensitivity to cognitive activities than deoxy-hemoglobin (Baker et al., 2018; Brockington et al., 2018), therefore we only analyzed and reported results for this variable. The part of the session analyzed only includes the concept generation phase, i.e., the whole session for the brainstorming technique (step 1 in Table 1), the second and last part of the session for the morphological technique (step 2 and 3 in Table 1) and the last part of the session for the TRIZ technique (step 4 in Table 1). Our analysis took a nonoverlapping window approach commonly used in cognitive design studies to equally divide the design process into ten segments (deciles) (Kan \& Gero, 2017). This non-overlapping approach normalized the concept generation sessions over time. $\mathrm{HbO}$ for each segment was averaged together for all participants to create an average $\mathrm{HbO}$ for each of the ten segments (or deciles). This type of averaging technique is common within cognitive neuroscience (Hu \& Shealy, 2019). To capture the transfer of high activation across the deciles, the highest activated node is established by averaging $\mathrm{HbO}$ values across subjects for each channel per decile.

\section{RESULTS}

\subsection{Different temporal activation of channels for brainstorming, morphological analysis and TRIZ}

The highest activated channel for brainstorming is situated in the right hemisphere at first (right VLPFC for deciles 1 and 4, right DLPFC for deciles 2 and 5) before switching between the mPFC and the left VLPFC for the following deciles, illustrated in Figure 2(a). In decile 10, the highest activation is found in the right DLPFC. Cognitive processes that recruit each sub-region are associated with 
previous findings (Milovanovic et al., 2020). The right part of the PFC is known to be recruited to explore the problem space (right VLPFC) and to support divergent thinking (right DLPFC) (AzizZadeh et al., 2013; Goel \& Grafman, 2000; Goel \& Vartanian, 2005; Wu et al., 2015).

Examining the channel activation for morphological analysis (Figure 2(b)), higher activation in the right hemisphere in the first half of the session (deciles 1 to 4) might suggest divergent thinking and exploration of the problem space (Aziz-Zadeh et al., 2013; Goel \& Grafman, 2000; Wu et al., 2015). Higher activation then appears (deciles 5 and 6) in the medial part of the PFC. This might be associated with cognitive processes related to adaptive decision-making (Euston et al., 2012). Towards the end of the session, high activation is situated in the left side of the PFC (deciles 7 to 9). This region of the brain is generally associated with goal-oriented planning and analytical judgment (Aziz-Zadeh et al., 2013). In the last decile, the higher activation appears in the right VLPFC. The right VLPFC is generally associated with problem identification (Aziz-Zadeh et al., 2009).

The highest activated node for TRIZ is always situated either in the mPFC (deciles 3, 4 and 5), a region generally associated with memory retrieval and adaptive decision-making (Euston et al., 2012), or the left part of the PFC for the other 7 deciles. This is a region of the brain generally associated with supporting rule-based design, goal-directed planning, and analytical judgment (Figure 2(c)) (Aziz-Zadeh et al., 2013). This trend only appears for TRIZ.

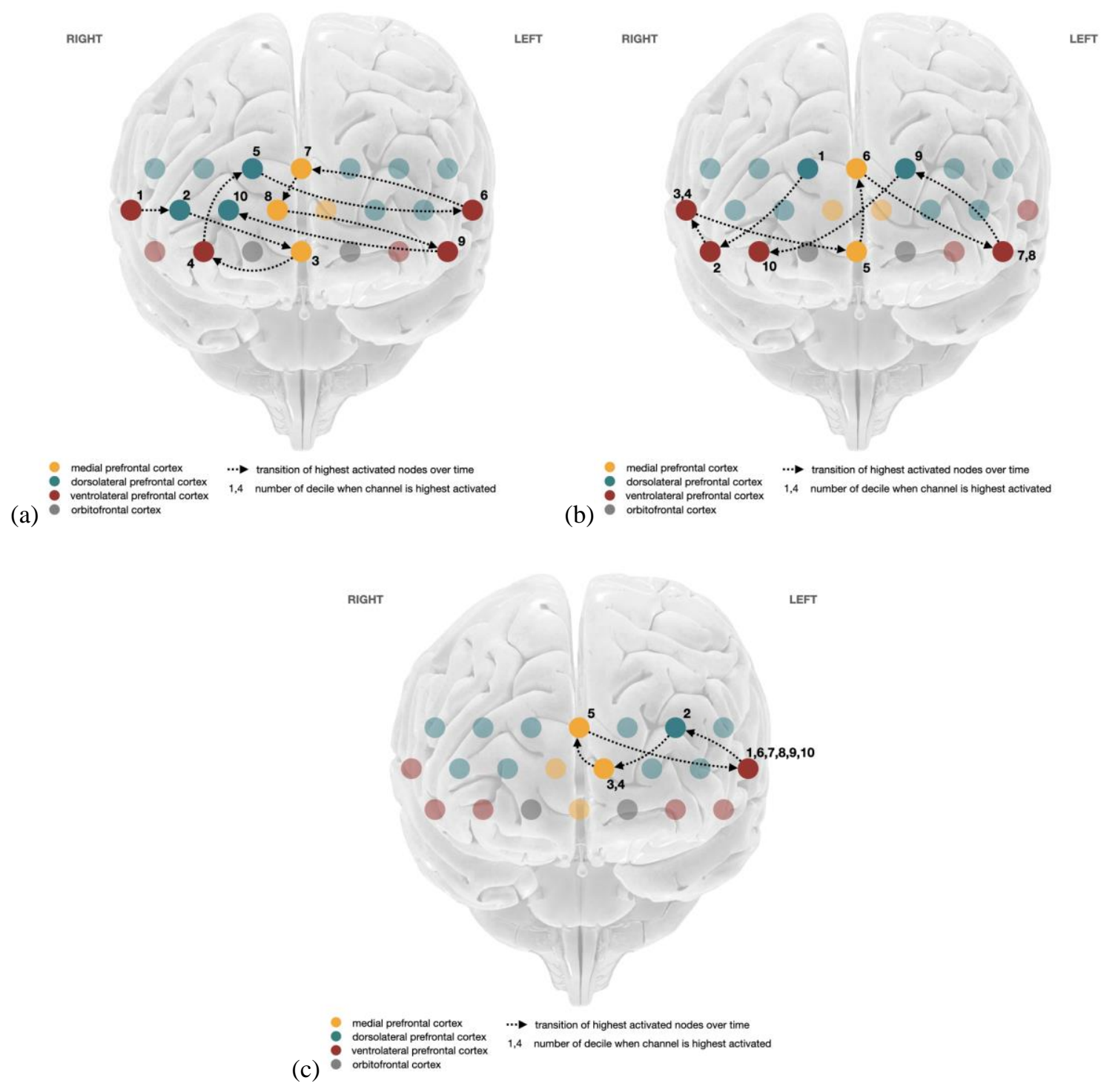

Figure 2. Transition paths of highest activated channels across time for (a) brainstorming,

(b) morphological analysis and (c)TRIZ. The black numbers refer to the time deciles; the colored circles refer to the channels. (Base of brain image copyright (C) Society for Neuroscience (2017)) 


\subsection{Neuro-activation patterns suggest different cognitive patterns}

Figure 3 represents a timeline of the cognitive functions defined in the literature (Table 2) associated with the highly activated sub-regions of the PFC for brainstorming, Figure 3(a), morphological analysis, Figure 3(b) and TRIZ, Figure 3(c). The potential neurophysiological association for brainstorming and morphological analysis suggests that participants may have started the session by evaluating the design problem, generating alternative hypothesis to explore the problem space and relied on visuo-spatial divergent thinking to creatively address the design problem. This first phase is followed by activation in the medial PFC, which is generally associated with adaptative decisionmaking based on memory retrieval, appearing in the $7^{\text {th }}$ and $8^{\text {th }}$ deciles for brainstorming (Figure 3(a)) and the $5^{\text {th }}$ and $6^{\text {th }}$ deciles for morphological analysis (Figure 3(b)). In the last part of the morphological analysis session, participants may have engaged in rule-based design and goal-directed planning because regions of their brain most activated are generally associated with these cognitive functions (Aziz-Zadeh et al., 2013; Gabora, 2010). Temporal neurophysiological activation patterns for TRIZ stand out compared to the observed neurophysiological patterns for the other two techniques. These patterns suggest an association with rule-based design while starting the concept generation phase. Then, high activation switch to the MPFC generally associated with adaptative decision-making process based on memory retrieval. During the second half of the session, the highest activated region is associated with goal-directed planning (Figure 3(c)).
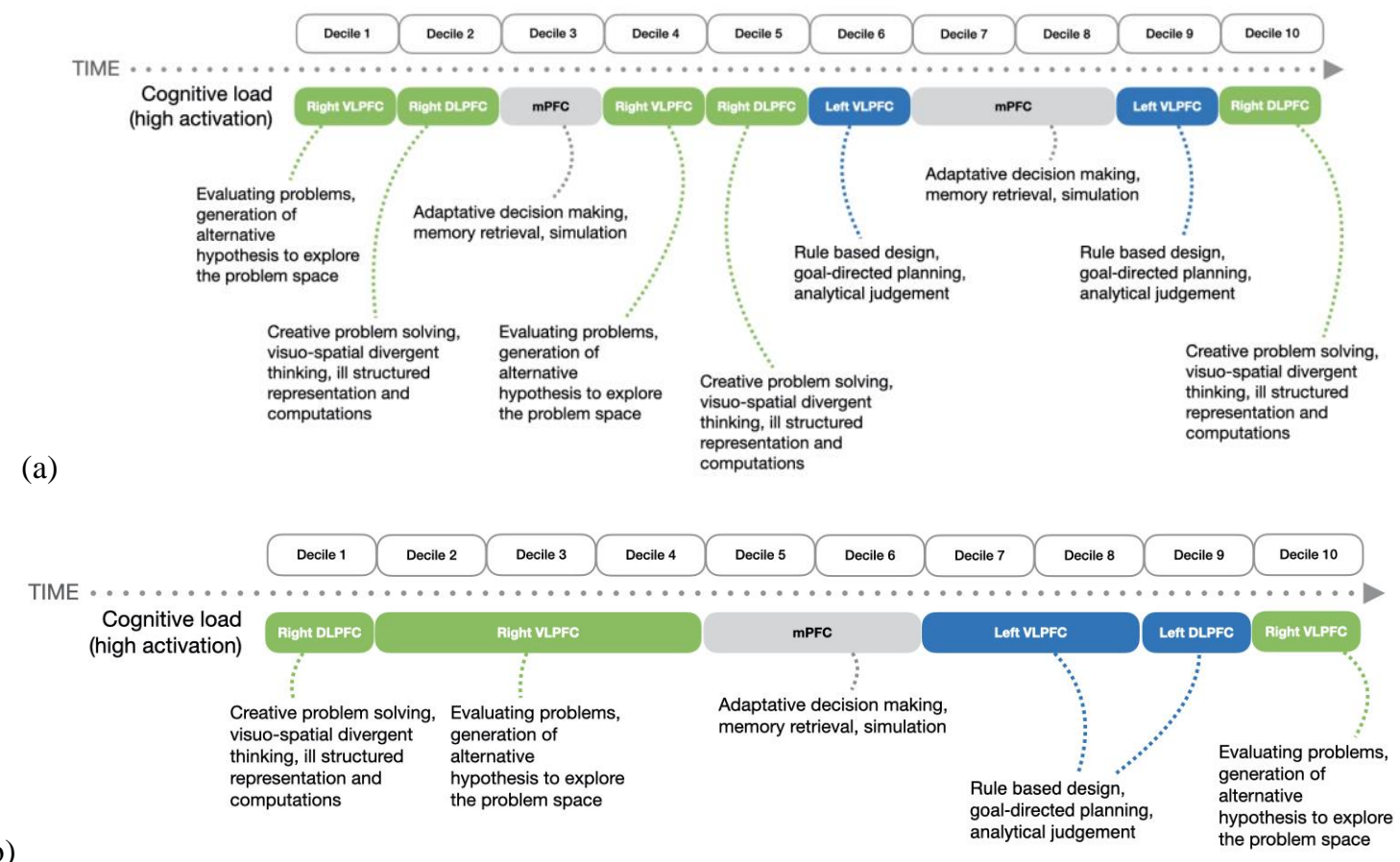

(b)

(c)

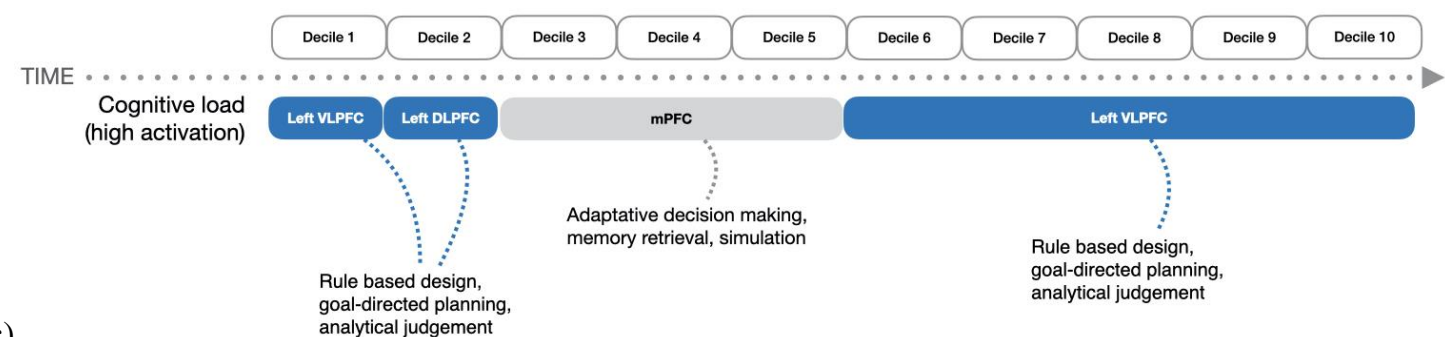

Figure 3. Concept generation timeline showing PFC highly activated sub-regions (cognitive load) for (a) brainstorming, (b) morphological analysis and (c)TRIZ. Color blue indicates sub-regions of the PFC in the left hemisphere, color grey indicates sub-regions of the PFC in the medial part and color green indicates sub-regions of the PFC in the right hemisphere. Associated cognitive functions are based on previous work (see Table 2). 


\section{DISCUSSION}

\subsection{Activation of the MPFC for all types of concept generation techniques}

The findings point towards specific temporal dynamics of neurophysiological activation in the prefrontal cortex (PFC) for each concept generation technique. The medial PFC (mPFC) is highly activated when generating concepts regardless of the problem structuredness but at different moments in time. The $\mathrm{mPFC}$ is believed to be an essential region for neural networks relevant for socio-emotional processing, such as cognitive empathy and perspective taking (Seitz et al., 2006). Given that three similar design problems asked participants to design products for disadvantaged groups (i.e., the elderly, the hearing impaired, and the blind), this consistently activated region might suggest processing of cognitive empathy when generating concepts. Prior neuroscience literature also suggests the mPFC is recruited in memory retrieval and association learning (Euston et al., 2012). Another possible explanation for the recruitment of this region is that students cognitively made associations between ideas during the concept generation process. The activation of the mPFC also relates to the ability to simulate future imaginative events (Meyer et al., 2019). Students rely on their analysis and understanding of the design problem to propose ideas. The high recruitment of the mPFC aligns with the task at hand as students engage in adaptive decision making to imagine an artefact and simulate usage.

\subsection{Brain behavior in the PFC is similar using brainstorming and morphological analysis techniques for the first half of the session}

During brainstorming, higher activation in the right part of the PFC dominates for the first half of the session. Brainstorming is usually processed with an intuitive exploration of solution space, and students tend to rely on divergent thinking to generate multiple concepts (Gero et al., 2013). Divergent thinking is usually related to activation in the right PFC (Aziz-Zadeh et al., 2013; Goel \& Grafman, 2000; Kleibeuker et al., 2013; Wu et al., 2015), which is congruent with our findings in the first half of the brainstorming session. Both the right ventrolateral PFC (VLPFC) and right dorsolateral PFC (DLPFC) dominate in high activation at the beginning of the brainstorming session. The right DLPFC relates to illstructured representation and computations (Goel \& Grafman, 2000) and the right VLPFC is known to be recruited for evaluating problems rather than solving them (Aziz-Zadeh et al., 2009) and generating alternative hypotheses (Goel \& Vartanian, 2005). Previous findings align with ours, as students started the brainstorming session without prior problem structuring, therefore had to put cognitive effort into finding missing information about the problem and exploring the problem space.

Similar to brainstorming, high activation during the first half of the morphological analysis session is centered in the right part of the PFC, in the VLPFC and DLPFC. In this case, the problem was pre-structured, since students spent time to decompose the problem into sub-problems before engaging in concept generation. Higher activation in the right PFC in the first half of the morphological analysis session might indicate that students needed to put effort into evaluating and synthesizing sub-problems to generate concepts.

In contrast, cognitive activation in the first half of the TRIZ session appears predominantly in the left part of the PFC (dorsolateral and ventrolateral), a region of the brain generally associated with rule-based design and goal-directed planning of novel solutions (Aziz-Zadeh et al., 2013; Ruh et al., 2012). Activation in the medial part of the PFC related to cognitive empathy, adaptative decision-making, memory retrieval, and situated associations (Euston et al., 2012; Seitz et al., 2006) is also dominant when using the morphological analysis technique. During TRIZ, students spent time identifying and formulating the problem, and searching for catalogued design solutions before developing concepts which can explain a lower cognitive effort put into problem exploration and evaluation based on activation in the VLPFC and DLPFC. When using unstructured (brainstorming) and semi-structured (morphological analysis), participants displayed a similar brain activation pattern over time for the first half of the session.

\subsection{Brain behavior in the PFC is similar using morphological analysis and TRIZ techniques for the second half of the session}

During the second half of the session, brain activation during brainstorming is dominant in the mPFC. The left VLPFC and the right DLPFC are activated more than other sub-regions towards the end of the session. Increased activation in the right and left prefrontal cortex is generally observed during brainstorming in other studies (e.g., see Heilman et al., 2003). Our results match with these previous study findings. Increased activation in the $\mathrm{mPFC}$ at different times depending on the technique, might 
suggest students have engaged in an adaptative decision-making (Euston et al., 2012). This is followed by alternating high activation between regions of the brain in the left and right side of the PFC, generally associated with rule-based design and divergent thinking (Aziz-Zadeh et al., 2013). In contrast, when using the goal-directed techniques like morphological analysis and TRIZ (Taura \& Nagai, 2013) in the second half of the concept generation session, the left hemisphere is recruited more than for brainstorming. This result is consistent with prior design cognition research that concludes the function of analytical judgments, convergent thinking, and goal-directed planning recruit activation in the left hemisphere, and specifically the left DLPFC (Aziz-Zadeh et al., 2009). Using morphological analysis and TRIZ, students had already spent cognitive effort in exploring and reframing the problem space; therefore, it was expected that more effort would be put into convergent thinking and developing a solution. When using structured (TRIZ) and semi-structured (morphological analysis), participants displayed a similar brain activation pattern over time for the second half of the session.

\section{CONCLUSION}

The results uncover consistent activation in the medial prefrontal cortex (PFC) when using all three techniques. The recruitment of activation in this region of the brain was previously associated with cognitive empathy and memory association during concept generation. Additional highly activated sub-regions of the PFC during brainstorming (unstructured design problem) and morphological analysis (semi-structured) are more frequent in the right hemisphere. The right hemisphere of the PFC is generally associated with divergent thinking. When using TRIZ (structured design problem), the left part of the PFC is in higher demand compared to the right part of the PFC. With an increased in problem structuring, activation switches from the medial right to the medial left part of the PFC. These changes are likely to impact how information is transmitted within regions of the PFC and other parts of the brain. This could be related to the structured dual processing between divergent and convergent thinking that concept generation requires (Goldschmidt, 2016). The dynamics in activation of subregions between techniques could reflect a sequential variation of cognitive processes related to the level of structuredness of the design problem.

These results also demonstrate that brain imaging methods such as fNIRS are suitable for design neurocognition research. The findings are based on an interpretation of cognitive functions associated to sub-region activation in the PFC. More research is required to strengthen the relationship between cognitive and neurocognitive results. Future work should begin to tackle cognition and neurocognition through the concurrent analysis of verbal protocols of designers to infer their cognitive processes and of neurophysiological signals accounting for designers' brain activation (Pike et al., 2014). Carrying out think-aloud protocols while brain imaging offers the potential to connect more directly design cognition from the coding of the protocols with the brain activations invoked by those cognitive acts. By correlating the design quality and neurocognitive performance, we can start to decipher how patterns of neuro-activation correspond to better design. The participants were students from the same engineering background and with limited design experience. The findings could represent brain behaviors of novices not experts and could be domain related. Future work should integrate expert designers from different backgrounds to explore if their design neurocognition differs from what was observed in this study. Gaining more understanding of designers' minds and brains while designing will open avenues for the development of new tools to assist designing and enhance creativity.

\section{ACKNOWLEDGEMENT}

This material is based in part on work supported by The National Science Foundation, through Grant EEC-1929892 and EEC-1929896. Any opinions, findings and conclusions or recommendations expressed in this material are those of the author(s) and do not necessarily reflect the views of the National Science Foundation.

\section{REFERENCE}

Alexiou, K., Zamenopoulos, T., \& Gilbert, S. (2011). Imaging the designing brain: A neurocognitive exploration of design thinking. In J. S. Gero (Ed.), Design Computing and Cognition '10 (pp. 489-504). Springer Netherlands. https://doi.org/10.1007/978-94-007-0510-4_26

Altshuller, G. (1997). 40 Principles: TRIZ Keys to Technical Innovation (Technical Innovation Center, INC.). 
Aziz-Zadeh, L., Kaplan, J. T., \& Iacoboni, M. (2009). “Aha!”: The neural correlates of verbal insight solutions. Human Brain Mapping, 30(3), 908-916. https://doi.org/10.1002/hbm.20554

Aziz-Zadeh, L., Liew, S.-L., \& Dandekar, F. (2013). Exploring the neural correlates of visual creativity. Social Cognitive and Affective Neuroscience, 8(4), 475-480. https://doi.org/10.1093/scan/nss021

Baker, J. M., Bruno, J. L., Gundran, A., Hosseini, S. M. H., \& Reiss, A. L. (2018). FNIRS measurement of cortical activation and functional connectivity during a visuospatial working memory task. PLOS ONE, 13(8), e0201486. https://doi.org/10.1371/journal.pone.0201486

Borgianni, Y., \& Maccioni, L. (2020). Review of the use of neurophysiological and biometric measures in experimental design research. Artificial Intelligence for Engineering Design, Analysis and Manufacturing, 1-38. https://doi.org/10.1017/S0890060420000062

Brockington, G., Balardin, J. B., Zimeo Morais, G. A., Malheiros, A., Lent, R., Moura, L. M., \& Sato, J. R. (2018). From the laboratory to the classroom: The potential of functional Near-Infrared Spectroscopy in educational neuroscience. Frontiers in Psychology, 9, 1840. https://doi.org/10.3389/fpsyg.2018.01840

Chulvi, V., Sonseca, Á., Mulet, E., \& Chakrabarti, A. (2012). Assessment of the relationships among design methods, design activities, and creativity. Journal of Mechanical Design, 134(11), 111004. https://doi.org/10.1115/1.4007362

Dietrich, A. (2004). The cognitive neuroscience of creativity. Psychonomic Bulletin \& Review, 11(6), 10111026. https://doi.org/10.3758/BF03196731

Euston, D. R., Gruber, A. J., \& McNaughton, B. L. (2012). The role of medial prefrontal cortex in memory and decision-making. Neuron, 76(6), 1057-1070. https://doi.org/10.1016/j.neuron.2012.12.002

Fink, A., Grabner, R. H., Benedek, M., Reishofer, G., Hauswirth, V., Fally, M., Neuper, C., Ebner, F., \& Neubauer, A. C. (2009). The creative brain: Investigation of brain activity during creative problem solving by means of EEG and FMRI. Human Brain Mapping, 30(3), 734-748. https://doi.org/10.1002/hbm.20538

Fu, K. K., Sylcott, B., \& Das, K. (2019). Using fMRI to deepen our understanding of design fixation. Design Science, 5, e22. https://doi.org/10.1017/dsj.2019.21

Gabora, L. (2010). Revenge of the "Neurds": Characterizing creative thought in terms of the structure and dynamics of memory. Creativity Research Journal, 22(1), 1-13. https://doi.org/10.1080/10400410903579494

Gero, J. S. (1999). A new model of designing which includes its situatedness, in J. Gu and Z. Wei (eds), CAADRIA'99, Shanghai Scientific and Technological Literature Publishing House, Shanghai, China, pp. 235-242.

Gero, J. S., Jiang, H., \& Williams, C. B. (2013). Design cognition differences when using unstructured, partially structured, and structured concept generation creativity techniques. International Journal of Design Creativity and Innovation, 1(4), 196-214. https://doi.org/10.1080/21650349.2013.801760

Gero, J. S., \& Milovanovic, J. (2020). A framework for studying design thinking through measuring designers' minds, bodies and brains. Design Science, 6(e19). https://doi.org/10.1017/dsj.2020.15

Gilbert, S. J., Zamenopoulos, T., Alexiou, K., \& Johnson, J. H. (2010). Involvement of right dorsolateral prefrontal cortex in ill-structured design cognition: An fMRI study. Brain Research, 1312, 79-88. https://doi.org/10.1016/j.brainres.2009.11.045

Goel, V. (2014). Creative brains: Designing in the real world. Frontiers in Human Neuroscience, 8. https://doi.org/10.3389/fnhum.2014.00241

Goel, V., \& Grafman, J. (2000). Role of the right prefrontal cortex in ill-structured planning. Cognitive Neuropsychology, 17(5), 415-436. https://doi.org/10.1080/026432900410775

Goel, V., \& Vartanian, O. (2005). Dissociating the roles of right ventral lateral and dorsal lateral prefrontal cortex in generation and maintenance of hypotheses in set-shift problems. Cerebral Cortex, 15(8), 1170 1177. https://doi.org/10.1093/cercor/bhh217

Goldschmidt, G. (2016). Linkographic evidence for concurrent divergent and convergent thinking in creative design. Creativity Research Journal, 28(2), 115-122. https://doi.org/10.1080/10400419.2016.1162497

Goucher-Lambert, K., Moss, J., \& Cagan, J. (2019). A neuroimaging investigation of design ideation with and without inspirational stimuli-Understanding the meaning of near and far stimuli. Design Studies, 60, 1-38. https://doi.org/10.1016/j.destud.2018.07.001

Hay, L., Duffy, A. H. B., Gilbert, S. J., Lyall, L., Campbell, G., Coyle, D., \& Grealy, M. A. (2019). The neural correlates of ideation in product design engineering practitioners. Design Science, 5, e29. https://doi.org/10.1017/dsj.2019.27

Hay, L., Duffy, A. H. B., McTeague, C., Pidgeon, L. M., Vuletic, T., \& Grealy, M. (2017). A systematic review of protocol studies on conceptual design cognition: Design as search and exploration. Design Science, 3. https://doi.org/10.1017/dsj.2017.11

Heilman, K. M., Nadeau, S. E., \& Beversdorf, D. O. (2003). Creative innovation: Possible brain mechanisms. Neurocase, 9(5), 369-379. https://doi.org/10.1076/neur.9.5.369.16553

Hu, M., \& Shealy, T. (2019). Application of functional Near-Infrared Spectroscopy to measure engineering decision-making and design cognition: Literature review and synthesis of methods. Journal of Computing in Civil Engineering, 33(6), 04019034. https://doi.org/10.1061/(ASCE)CP.1943-5487.0000848 
Kan, J. W., \& Gero, J. S. (2017). Quantitative Methods for Studying Design Protocols. Springer Netherlands. https://doi.org/10.1007/978-94-024-0984-0

Kleibeuker, S. W., Koolschijn, P. C. M. P., Jolles, D. D., Schel, M. A., De Dreu, C. K. W., \& Crone, E. A. (2013). Prefrontal cortex involvement in creative problem solving in middle adolescence and adulthood. Developmental Cognitive Neuroscience, 5, 197-206. https://doi.org/10.1016/j.dcn.2013.03.003

Lara, A. H., \& Wallis, J. D. (2015). The role of prefrontal cortex in working memory: A mini review. Frontiers in Systems Neuroscience, 9. https://doi.org/10.3389/fnsys.2015.00173

Laspia, A., Montagna, F., \& Törlind, P. (2019). Contrasting divergent and convergent thinking by electroencephalography and eye tracking. In A. Chakrabarti (Ed.), Research into Design for a Connected World (Vol. 1, pp. 179-188). Springer Singapore. https://doi.org/10.1007/978-981-13-5974-3_16

Luft, C. D. B., Zioga, I., Banissy, M. J., \& Bhattacharya, J. (2017). Relaxing learned constraints through cathodal tDCS on the left dorsolateral prefrontal cortex. Scientific Reports, 7(1), 2916. https://doi.org/10.1038/s41598-017-03022-2

Meyer, M. L., Hershfield, H. E., Waytz, A. G., Mildner, J. N., \& Tamir, D. I. (2019). Creative expertise is associated with transcending the here and now. Journal of Personality and Social Psychology, 116(4), 483494. https://doi.org/10.1037/pspa0000148

Milovanovic, J., Hu, M., Shealy, T., \& Gero, J. S. (2020). Evolution of brain network connectivity in the prefrontal cortex during concept generation using brainstorming for a design task. ASME 2020 International Design Engineering Technical Conferences and Computers and Information in Engineering Conference. https://doi.org/10.1115/DETC2020-22563

Pike, M. F., Maior, H. A., Porcheron, M., Sharples, S. C., \& Wilson, M. L. (2014). Measuring the effect of think aloud protocols on workload using fNIRS. Proceedings of the SIGCHI Conference on Human Factors in Computing Systems, 3807-3816. https://doi.org/10.1145/2556288.2556974

Ruh, N., Rahm, B., Unterrainer, J. M., Weiller, C., \& Kaller, C. P. (2012). Dissociable stages of problem solving (II): First evidence for process-contingent temporal order of activation in dorsolateral prefrontal cortex. Brain and Cognition, 80(1), 170-176. https://doi.org/10.1016/j.bandc.2012.02.012

Santosa, H., Aarabi, A., Perlman, S. B., \& Huppert, T. J. (2017). Characterization and correction of the falsediscovery rates in resting state connectivity using functional near-infrared spectroscopy. Journal of Biomedical Optics, 22(5), 055002. https://doi.org/10.1117/1.JBO.22.5.055002

Schön, D. (1983). The reflective practitioner: How professionals think in action. Temple Smith.

Seitz, R. J., Nickel, J., \& Azari, N. P. (2006). Functional modularity of the medial prefrontal cortex: Involvement in human empathy. Neuropsychology, 20(6), 743-751. https://doi.org/10.1037/0894-4105.20.6.743

Shealy, T., Hu, M., \& Gero, J. S. (2018). Patterns of cortical activation when using concept generation techniques of brainstorming, morphological analysis, and TRIZ. In A. International (Ed.), Design Engineering Technical Conferences and Computers and Information in Engineering Conference. Quebec.

Shealy, T., \& Gero, J. S. (2019). The neurocognition of three engineering concept generation techniques. Proceedings of the Design Society: International Conference on Engineering Design, 1(1), 1833-1842. https://doi.org/10.1017/dsi.2019.189

Shealy, T., Gero, J., Hu, M., \& Milovanovic, J. (2020). Concept generation techniques change patterns of brain activation during engineering design. Design Science, 6. https://doi.org/10.1017/dsj.2020.30

Shealy, T., Grohs, J., Hu, M., Maczka, D., \& Panneton, R. (2017). Investigating design cognition during brainstorming tasks with freshmen and senior engineering students using functional Near Infrared Spectroscopy. ASEE Annual Conference, Columbus, OH.

Smith, G. F. (1998). Idea-generation techniques: A formulary of active ingredients. The Journal of Creative Behavior, 32(2), 107-134. https://doi.org/10.1002/j.2162-6057.1998.tb00810.x

Society for Neuroscience: https://www.brainfacts.org/3d-brain (accessed: 8 November 2020)

Tang, H.-H., Chen, Y.-L., \& Gero, J. S. (2011). The influence of design methods on the design process: Effect of use of scenario, brainstorming, and synectics on designing. Design Research Society, Bangkok, Thailand.

Taura, T., \& Nagai, Y. (2013). A systematized theory of creative concept generation in design: First-order and high-order concept generation. Research in Engineering Design, 24(2), 185-199. https://doi.org/10.1007/s00163-013-0152-6

Vieira, S., Gero, J. S., Delmoral, J., Gattol, V., Fernandes, C., Parente, M., \& Fernandes, A. A. (2020). The neurophysiological activations of mechanical engineers and industrial designers while designing and problem-solving. Design Science, 6, e26. https://doi.org/10.1017/dsj.2020.26

Wu, X., Yang, W., Tong, D., Sun, J., Chen, Q., Wei, D., Zhang, Q., Zhang, M., \& Qiu, J. (2015). A metaanalysis of neuroimaging studies on divergent thinking using activation likelihood estimation: Divergent Thinking. Human Brain Mapping, 36(7), 2703-2718. https://doi.org/10.1002/hbm.22801 